\title{
Existence and Uniqueness of Solutions in Some Boundary Conditions for Fractional Differential Systems
}

\author{
Münevver Tuz ${ }^{1, *}$ \\ 1,* Firat University, Faculty of Science, Departmant of Mathematics, Elazığ, Turkey, (ORCID: 0000-0002-9620-247X), mtuz@firat.edu.tr
}

(1st International Conference on Applied Engineering and Natural Sciences ICAENS 2021, November 1-3, 2021)

(DOI: 10.31590/ejosat.1021579)

ATIF/REFERENCE: Tuz, M. (2021). Existence of Solutions for Fractional Differential Systems with Periodic and Anti-periodic Boundary Conditions.European Journal of Science and Technology, (28), 1486-1491.

\begin{abstract}
In this article, fractional case of periodic problems is discussed. Considering the time fractional heat equation, inverse problems with periodic and anti-periodic boundary conditions were created. For these problems, the Fourier method was used to obtain existence and uniqueness results. The fractional derivative of a periodic function was analyzed along the real axis, and the periodic behavior of linear systems in case of fractions was investigated.
\end{abstract}

Keywords: Periodic, Anti-periodic, Fractional Derivative, Fourier Method, Linear System.

\section{Kesirli Diferansiyel Sistemler için Verilen Bazı Sınır Koşullarında Çözümlerin Varlığı ve Tekliği}

$\ddot{O} \mathbf{z}$

Bu makalede, periyodik problemlerin kesirli durumu tartışılmaktadır. Zaman kesirli ısı denklemi göz önünde bulundurularak, periyodik ve anti-periyodik sınır koşulları ile ters problemler oluşturulmuştur. Bu problemler için varlık ve teklik sonuçlarını elde etmek için Fourier yöntemi kullanılmıştır. Periyodik bir fonksiyonun kesirli türevi gerçek eksen boyunca analiz edilmiş ve lineer sistemlerin kesirler durumundaki periyodik davranışı araştırılmıştır.

Anahtar Kelimeler: Periyodik, Anti-periyodik, Kesirli Türev, Fourier Metodu, Lineer Sistem.

\footnotetext{
* Corresponding Author: mtuz@,firat.edu.tr
} 


\section{Introduction}

The advantages of fractional derivatives in science are that they have more flexibility in the model and provide a unique way for reality to emerge. Because when modeling a physical situation, it is essential not only to depend on the current time, but also to the past of the previous time, that is, the calculation of the fractional derivative of time at a time affects all previous processes with memory and hereditary.

Periodicity of functions constitutes an important situation in physics, technology and other scientific fields (Al-Mdallal, 2009). The influence of periodic functions on linear systems is well known (Ibrahim, 2007). This is true not only for integer systems, but also for fractions (Delavari et al., 2012). It has been proven in some studies that systems defined by some special fractional derivatives cannot have periodic solutions (Bouchaud, 1990 and Podlubny, 1999). However, the existence of periodic results obtained in linear systems has been characterized as a remarkable feature (Metzler, 2002). In the studies carried out so far, the solution of these two problems has still not been clarified. The problem that needs to be examined for an FD is, First, does a periodic signal of FD form a periodic function? The other, what is the relationship between the fractionalness of the linear system and its periodicity?We will consider them in this study. First of all, we give the Fourier series and its properties, as it is related to periodicity, and explain frequently used FD definitions. We show that we can easily obtain periodic signals if the derivative is defined only in R.For the other case, we obtain that systems can only have causal periodic data results if they are integer ordinal.To reach these studies, we will first base some tempered distributions (Mainardi, 2010).

When causality is applied to systems, causal systems result in zero at values where the variable is negative. This situation leads to a troublesome process. To turn this process into a positive step, the Heaviside unit step can be used. Thus, we consider the definition of "causal periodic", which makes a connection with a periodic data. In the light of these considerations, the problem of periodic functions and their derivatives, which forms the basis of the article, has been dealt with and solution steps have been created.

\section{Fourier Method and its Application}

In this study, the time fractional heat equation with an involution type periodic term is discussed. We want to obtain existence and uniqueness results for these problems with the Fourier method by creating a periodic and antiperiodic inverse problem.

\subsection{Periodic Functions and Their Relation to Derivatives}

This section explains the basic concepts of periodicity. In the first part, the periodic functions and the steps to solve the problems are determined and the necessary methods are discussed. For this, the Fourier transform and its properties are given. In the next section, the fractional derivatives of the periodic functions and their solutions are given step by step.

Definition. The definition of the periodic function is as follows,

$x_{p}(t)$, by an identity such that

$x_{p}(t)=x_{p}(t+T), \quad t \in R, T \in R^{+}$,

where $\mathrm{t}$ represents time, T represents period (Samko, 2003).

Corollary. Let $x_{b}(t)=x_{p}(t), t \in(a, b)$ with $b-a=T$.

The truth of the following equation is well known.

$x_{p}(t)=\sum_{n=-\infty}^{\infty} x_{b}(t-n T), t_{n}=n T, n \in N$

Definition. The following series can be written, with the definition being, $c(t), t \in R$,

$c(t)=\sum_{n=-\infty}^{\infty} \delta(t-n T)$

The convergence of the $\sum_{n=-\infty}^{\infty} \delta(t-n T)$ series is shown (Pooseh et al., 2011). If we choose delta like this,

$x_{b}(t)=x_{p}(t) * \sum_{n=-\infty}^{\infty} \delta(t-n T)$

* is called the convolution operation and corresponds to

$$
(f * g)(t)=\int_{-\infty}^{\infty} f(\tau) g(t-\tau) d \tau .
$$

To calculate fractional derivatives, we start from the linearity of its operators. We try to calculate the solution by taking the Eq. (2) into consideration and obtaining the terms of fractional derivatives,

$$
x_{p}{ }^{(\alpha)}(t)=\sum_{n=-\infty}^{\infty} x_{b}{ }^{(\alpha)}(t)(t-n T)
$$

From the definition of convolution,

$$
x_{p}{ }^{(\alpha)}(t)=x_{b}{ }^{(\alpha)}(t) * \sum_{n=-\infty}^{\infty} \delta(t-n T)
$$

The significance of this approach is that it specifies a function on which only derivatives defined will be useful. The point to note here is that although $x_{b}(\mathrm{t})$ is finite, the derivative of $x_{b}(\mathrm{t})$ can no longer be expected to be finite. This can be proved by important fractional derivatives formulations such as Grünwald-Lenikov, Riemann-Liouville, Liouville, Caputo (Podlubny, 1999). 


\subsection{Fourier Method} follows,

We define the Fourier transform using the shift property as

$C(\omega)=\sum_{n=-\infty}^{\infty} \delta(t-n T) e^{i n \omega t}$

where $\omega$ is the angular frequency. If taken as

$C(\omega)=F\{c(t)\}$, It can be called the Fourier series

$C(\omega)=\sum_{n=-\infty}^{\infty} \delta(\omega-n \tau)$

where $\tau T=2 \pi$ is (Bochner, 1949). If the function is a periodic function with a period $\mathrm{T}$, the Fourier transform is also periodic and has a period

$\tau=T / 2 \pi$. If we combine the Fourier transform and convolution, we get

$$
\begin{array}{r}
X_{p}(\omega)=X_{b}(\omega) \frac{T}{2 \pi} \sum_{n=-\infty}^{\infty} \delta(\omega-n \tau) \\
=\frac{T}{2 \pi} \sum_{n=-\infty}^{\infty} X_{b}(\omega) \delta(\omega-n \tau) .
\end{array}
$$

For some functions $\mathrm{f}$, it gives and from here $f(t) \delta(t-$ $a)=f(a) \delta(t-a)$ and we deduce

$X_{p}(\omega)=2 \pi \frac{T}{2 \pi} \sum_{n=-\infty}^{\infty} X_{b}(n \tau) \delta(\omega-n \tau)$

and, by inversion

$x_{p}(t)=\sum_{n=-\infty}^{\infty} X_{b}(n \tau) e^{-i n \frac{2 x}{T} t}$.

its terar with the same periods is represented by the Fourier series, and is expressed as a series (7). The resulting Fourier series defines a function that is periodic in the whole real axis (Tapdigoglu, 2019).

In our study based on the Fourier method, we will consider the relationship we will establish between the perturbative term of the evolution type according to the space variable and the heat equation as an inverse problem.

Let's define the heat equation as

$D_{t}^{\alpha} u(x, t)-u_{x x}(x, t)+\varepsilon u_{x x}(1-x, t)=f(x)$,

for $(x, t) \in \Omega=\{0<x<1,0<t<T<\infty\}, 0<\alpha<1$ where $D_{t}^{\alpha}$ is the Caputo derivative and $\varepsilon$ is a real number.

we will obtain $(u(x, t), f(x))$ which are smooth functions, we will satisfy Eq. (8) under the conditions given below,

$u(x, 0)=\varphi(x), x \in[0,1]$,

$u(x, T)=\psi(x), x \in[0,1]$ and periodic boundary conditions

$u(0, t)=u(1, t), t \in[0, T]$,

$u_{x}(0, t)=u_{x}(1, t)$,

anti-periodic boundary conditions

$u(0, t)=-u(1, t), t \in[0, T]$

$u_{x}(0, t)=-u_{x}(1, t)$

$(u(x, t), f(x))$, where $u \in C^{2}(\bar{\Omega})$ and $f \in C([0,1])$.

Now here we will use the Fourier method to solve the P and AP problems, with this application Eq. (8) poses a spectral problem.

For $\mathrm{P}$ and AP problems we use the following theorems.

Theorem. Let $|\varepsilon|<1$, where $\varphi, \psi \in \mathrm{C}^{3}[01]$ and where $\varphi^{(i)}(0)=\varphi^{(i)}(1), \varphi_{x}{ }^{(i)}(0)=\varphi_{x}{ }^{(i)}(1)$ and $\psi^{(i)}(0)=\psi^{(i)}(1), \psi_{x}{ }^{(i)}(0)=\psi_{x}{ }^{(i)}(1), i=0,1,2$. Then problem $\mathrm{P}$ has only one solution and can be written as,

$u(x, t)=\varphi(x)$

$+\sum_{k=0}^{\infty} \frac{\left(1-E_{\alpha, 1}\left(-(1-\varepsilon)\left(k+\frac{1}{2}\right)^{2} t^{\alpha}\right)\right) \sin \left(k+\frac{1}{2}\right) x}{\left(1-E_{\alpha, 1}\left(-(1-\varepsilon)\left(k+\frac{1}{2}\right)^{2} T^{\alpha}\right)\right)\left(k+\frac{1}{2}\right)^{2}}\left(\varphi_{1 k}^{(2)}-\psi_{1 k}^{(2)}\right)+$

$\sum_{k=1}^{\infty} \frac{\left(1-E_{\alpha, 1}\left(-(1+\varepsilon)(2 k)^{2} t^{\alpha}\right)\right) \sin k x}{\left(1-E_{\alpha, 1}\left(-(1+\varepsilon)(2 k)^{2} T^{\alpha}\right)\right)(2 k)^{2}}\left(\varphi_{2 k}^{(2)}-\psi_{2 k}^{(2)}\right)$,

$f(x)=-\varphi^{\prime \prime}(x)+\varepsilon \varphi^{\prime \prime}(1-x)$

$+\sum_{k=0}^{\infty} \frac{\left((1-\varepsilon)\left(\varphi_{1 k}^{(2)}-\psi_{1 k}^{(2)}\right)\right)}{\left(1-E_{\alpha, 1}\left(-(1-\varepsilon)\left(k+\frac{1}{2}\right)^{2} T^{\alpha}\right)\right)} \sin \left(k+\frac{1}{2}\right) x$

$+\sum_{k=1}^{\infty} \frac{\left((1+\varepsilon)\left(\varphi_{2 k}^{(2)}-\psi_{2 k}^{(2)}\right)\right)}{\left(1-E_{\alpha, 1}\left(-(1+\varepsilon)(2 k)^{2} T^{\alpha}\right)\right)} \sin k x$,

where

$\varphi_{1 k}^{(2)}=\left(\varphi^{\prime \prime}(x), y_{k+\frac{1}{2}}^{P}\right), \varphi_{2 k}^{(2)}=\left(\varphi^{\prime \prime}(x), y_{k}^{P}\right)$

$\psi_{1 k}^{(2)}=\left(\psi^{\prime \prime}(x), y_{k+\frac{1}{2}}^{P}\right), \psi_{2 k}^{(2)}=\left(\psi^{\prime \prime}(x), y_{k}^{P}\right)$,

and $E_{\alpha, \beta}(\lambda t)$ is the Mittag-Leffler type function,

$E_{\alpha, \beta}(z)=\sum_{m=0}^{\infty} \frac{z^{m}}{\Gamma(\alpha m+\beta)}$.

Theorem. Let $|\varepsilon|<1$, where $\varphi, \psi \in C^{3}[0,1] \quad$ and where $\varphi^{(i)}(0)=-\varphi^{(i)}(1), \quad \varphi_{x}{ }^{(i)}(0)=-\varphi_{x}{ }^{(i)}(1)$ and $\psi^{(i)}(0)=-\psi^{(i)}(1), \quad \psi_{x}{ }^{(i)}(0)=-\psi_{x}{ }^{(i)}(1), i=0,1,2$. Then the solution of problem AP exists, is unique and it can bewritten in the form 
$u(x, t)=\varphi(x)+\frac{t}{T}\left(\psi_{0}-\varphi_{0}\right)$

$+\sum_{k=1}^{\infty} \frac{\left(1-E_{\alpha, 1}\left(-(1-\varepsilon)(2 k)^{2} t^{\alpha}\right)\right) \cos k x}{\left(1-E_{\alpha, 1}\left(-(1-\varepsilon)(2 k)^{2} T^{\alpha}\right)\right)(4 k)^{2}}\left(\psi_{1 k}^{(2)}-\varphi_{1 k}^{(2)}\right)$

$+\sum_{k=0}^{\infty} \frac{\left(1-E_{\alpha, 1}\left(-(1+\varepsilon)\left(k+\frac{1}{2}\right)^{2} t^{\alpha}\right)\right) \cos \left(k+\frac{1}{2}\right) x}{\left(1-E_{\alpha, 1}\left(-(1+\varepsilon)\left(k+\frac{1}{2}\right)^{2} T^{\alpha}\right)\right)\left(k+\frac{1}{2}\right)^{2}}\left(\psi_{2 k}^{(2)}-\varphi_{2 k}^{(2)}\right)$,

$f(x)=-\varphi^{\prime \prime}(x)+\varepsilon \varphi^{\prime \prime}(1-x)$

$+\sum_{k=1}^{\infty} \frac{\left((1-\varepsilon)\left(\varphi_{1 k}^{(2)}-\psi_{1 k}^{(2)}\right)\right)}{\left(1-E_{\alpha, 1}\left(-(1-\varepsilon)(2 k)^{2} T^{\alpha}\right)\right)} \cos k x$

$+\sum_{k=0}^{\infty} \frac{\left((1+\varepsilon)\left(\varphi_{2 k}^{(2)}-\psi_{2 k}^{(2)}\right)\right)}{\left(1-E_{\alpha, 1}\left(-(1+\varepsilon)\left(k+\frac{1}{2}\right)^{2} T^{\alpha}\right)\right)} \cos \left(k+\frac{1}{2}\right) x$,

where

$\varphi_{0}=\left(\varphi_{0}, y_{0}^{A P}\right), \varphi_{1 k}^{(2)}=\left(\varphi^{\prime \prime}(x), y_{k}^{A P}\right), \varphi_{2 k}^{(2)}=$

$\left(\varphi^{\prime \prime}(x), y_{k+\frac{1}{2}}^{A P}\right)$,

$\psi_{0}=\left(\psi_{0}, y_{0}^{A P}\right), \psi_{1 k}^{(2)}=\left(\psi^{\prime \prime}(x), y_{k}^{A P}\right), \psi_{2 k}^{(2)}=$

$\left(\psi^{\prime \prime}(x), y_{k+\frac{1}{2}}^{A P}\right)$.

\subsection{Existence of Solution of Periodic and Anti- Periodic Problems}

We will first prove the problem P. It can be proved in a similar way that the solution of the AP problem also exists. The eigenvalues of the problem (8)- (11) and their corresponding eigenfunctions are as follows:

$\lambda_{k+\frac{1}{2}}^{P}=(1+\varepsilon) 2 k^{2}, k \in N$,

$\lambda_{k}^{P}=(1-\varepsilon) 2 k^{2}, k \in N_{0}$,

with the corresponding eigenfunctions

$y_{0}^{P}=1$,

$y_{k+\frac{1}{2}}^{P}=\sqrt{2} \sin (2 k x), k \in N$,

$y_{2 k}^{P}=\sqrt{2} \cos (2 k x), k \in N$,

The problem (8)-(12) has the following eigenvalues.

$\lambda_{k+\frac{1}{2}}^{A P}=(1+\varepsilon)\left(k+\frac{1}{2}\right)^{2}, k \in N_{0}$,

$\lambda_{k}^{A P}=(1-\varepsilon)\left(k+\frac{1}{2}\right)^{2}, k \in N_{0}$,

and the corresponding eigenfunctions $y_{2 k+1}^{A P}=\sqrt{\frac{2}{\pi}} \cos \left(k+\frac{1}{2}\right) x, k \in N_{0}$,

$y_{k}^{A P}=\sqrt{\frac{2}{\pi}} \sin \left(k+\frac{1}{2}\right) x, k \in N_{0}$.

The $\mathrm{P}$ problem creates an orthonormal basis in $\mathrm{L}^{2}(0,1)$, so the problem (8)- (11) is a self adjoint problem and corresponds to the solution function below.

$u(x, t)=\sum_{k=0}^{\infty} u_{k}(t) \sin \left(k+\frac{1}{2}\right) x+\sum_{k=1}^{\infty} v_{k}(t) \sin k x$

$f(x)=\sum_{k=0}^{\infty} f_{k}{ }^{1} \sin \left(k+\frac{1}{2}\right) x+\sum_{k=1}^{\infty} f_{k}^{2} \sin k x$

where $f_{k}{ }^{1}, f_{k}{ }^{2}, u_{k}(t), v_{k}(t)$ are unknown. If we substitute (11) and (12) in (8) here, we find the constants and functions as follows, $u_{k}(t), v_{k}(t)$ and the constants $f_{k}{ }^{1}, f_{k}{ }^{2}$ :

$D^{\alpha} u_{k}(t)+(1-\varepsilon)\left(k+\frac{1}{2}\right)^{2} u_{k}(t)=f_{k}{ }^{1}$,

$D^{\alpha} v_{k}(t)+(1+\varepsilon) 2 k^{2} v_{k}(t)=f_{k}^{2}$,

We obtain

$u_{k}(t)=\frac{f_{k}{ }^{1},}{(1-\varepsilon)\left(k+\frac{1}{2}\right)^{2}}+C_{1 k} E_{\alpha, 1}\left(-(1-\varepsilon)\left(k+\frac{1}{2}\right)^{2} t^{\alpha}\right)$,

$v_{k}(t)=\frac{f_{k}^{2}}{(1+\varepsilon) k^{2}}++C_{2 k} E_{\alpha, 1}\left(-(1+\varepsilon) 2 k^{2} t^{\alpha}\right)$,

$\mathrm{C}_{1 \mathrm{k}}, \mathrm{C}_{2 \mathrm{k}}, f_{k}{ }^{1}, f_{k}{ }^{2}$ are unknown constants.To find these constants, we consider the conditions (11). the coefficients of the expansions being $\varphi(x)$ and $\psi(x)$

$\varphi_{1 k}=\sqrt{2} \int_{0}^{1} \varphi(x) \sin \left(k+\frac{1}{2}\right) x d x$,

$\varphi_{2 k}=\sqrt{2} \int_{0}^{1} \varphi(x) \sin (k x) d x$,

$\psi_{1 k}=\sqrt{2} \int_{0}^{1} \psi(x) \sin \left(k+\frac{1}{2}\right) x d x$,

$\psi_{2 k}=\sqrt{2} \int_{0}^{1} \psi(x) \sin (k x) d x$,

first we find $\mathrm{C}_{1 \mathrm{k}}$,

$u_{k}(0)=\frac{f_{k}{ }^{1},}{(1-\varepsilon)\left(k+\frac{1}{2}\right)^{2}}+\mathrm{C}_{1 \mathrm{k}}=\varphi_{1 k}$,

$u_{k}(t)=\frac{f_{k}{ }^{1},}{(1-\varepsilon)\left(k+\frac{1}{2}\right)^{2}}+\mathrm{C}_{1 \mathrm{k}} E_{\alpha, 1}\left(-(1-\varepsilon)\left(k+\frac{1}{2}\right)^{2} T^{\alpha}\right)=\psi_{1 k}$,

$\varphi_{1 k}-\mathrm{C}_{1 \mathrm{k}}+\mathrm{C}_{1 k} E_{\alpha, 1}\left(-(1-\varepsilon)\left(k+\frac{1}{2}\right)^{2} T^{\alpha}\right)=\psi_{1 k}$. 
Later

$\mathrm{C}_{1 \mathrm{k}}=\frac{\varphi_{1 k}-\psi_{1 k}}{1-E_{\alpha, 1}\left(-(1-\varepsilon)\left(k+\frac{1}{2}\right)^{2} T^{\alpha}\right)}$.

It is represented as a fixed $f_{k}{ }^{1}$,

$$
f_{k}{ }^{1}=(1-\varepsilon)\left(k+\frac{1}{2}\right)^{2} \varphi_{1 k}-(1-\varepsilon)\left(k+\frac{1}{2}\right)^{2} C_{1 k} .
$$

Now we find $\mathrm{C}_{2 \mathrm{k}}$,

$v_{k}(0)=\frac{f_{k}{ }^{2}}{(1+\varepsilon) 2 k^{2}}++C_{2 \mathrm{k}}=\psi_{2 k}$,

$v_{k}(T)=\frac{f_{k}^{2},}{(1+\varepsilon) k^{2}}++\mathrm{C}_{2 \mathrm{k}} e^{-(1+\varepsilon) 2 k^{2} T}=\psi_{2 k}$,

$\psi_{2 \mathrm{k}}-\mathrm{C}_{2 \mathrm{k}}+\mathrm{C}_{2 \mathrm{k}} \mathrm{e}^{-(1+\varepsilon) 2 \mathrm{k}^{2} \mathrm{~T}}=\psi_{2 k}$.

later,

$$
\mathrm{C}_{2 k}=\frac{\varphi_{2 k}-\psi_{2 k}}{1-E_{\alpha, 1}\left(-(1+\varepsilon) 2 k^{2} T^{\alpha}\right)} .
$$

For fixed we found $f_{k}^{2}$,

$$
\begin{gathered}
f_{k}{ }^{1}=(1-\varepsilon)\left(k+\frac{1}{2}\right)^{2} \varphi_{1 k}-(1-\varepsilon)\left(k+\frac{1}{2}\right)^{2} \mathrm{C}_{1 k} . \\
u(x, t)=\varphi(x)
\end{gathered}
$$

$\left.+\sum_{k=0}^{\infty} \mathrm{C}_{1 k} E_{\alpha, 1}\left(-(1-\varepsilon)\left(k+\frac{1}{2}\right)^{2} t^{\alpha}\right)-1\right) \sin \left(k+\frac{1}{2}\right) x$

$\left.+\sum_{\mathrm{k}=1}^{\infty} \mathrm{C}_{2 \mathrm{k}} \mathrm{E}_{\alpha, 1}\left(-(1+\varepsilon) 2 \mathrm{k}^{2} \mathrm{t}^{\alpha}\right)-1\right) \sin k x$.

Therefore, this solution found is recorded as the solution of Eq. (8). Likewise, the solution of problem (9)- (11) is found in a similar way.

\section{Results and Discussion}

In response to the questions of whether the fractional derivatives of a periodic signal is also a periodic function or does the linear fractional system have periodic data, we saw in this study. If the derivative is defined only in $\mathrm{R}$, we can easily obtain periodic signals. For the second problem, we have seen that systems can only have periodic data results if they are integer order.

When causality is applied to systems, causal systems result in zero at values where the variable is negative. This situation causes a troublesome process. We want to turn this process into a positive step. Thus, considering the Heaviside unit step principle and the causal periodic definition, which makes the connection with a periodic data, we can evaluate the periodic data results in case the systems are integer or fractional order.

\section{Conclusions and Recommendations}

In this study, periodic functions are considered as fractions. Liouville and Caputo, which are fractional derivatives frequently used in the literature, have been used. It has been shown that the fractional derivative of a periodic function defined along the real axis is also periodic. It has also been proven that derivatives of these functions are not periodic in some cases. Also, fractional periodic behavior of linear systems is discussed. It has been shown that when certain derivatives are applied to such systems, their periodicity does not change. It is concluded that only integer ordered linear systems can have sinusoidal periodic oscillations.

The existence and uniqueness of the solution in fractional periodic and antiperiodic inverse problems has been proven by the Fourier method. The importance of this in the literature is that the existence theorem tells us that this problem has a solution to look for, it will prevent the loss of effort and time to be spent to find this special solution. It contributes to science in this respect.

\section{Acknowledge}

We would like to express our gratitude to the anonymous reviewers and editors for their valuable comments and suggestions which improve the quality of present paper.

\section{References}

Al-Mdallal, Q. M., (2009). An Efficient Method for Solving Fractional Sturm-Liouville Problems, Chaos Solitons Fractals, Vol.40, 183-189.

Boroomand, A., Menhaj, M.B., (2009). Fractional-Order Hopfield Neural Networks, Advances in Neuro-Information Processing, 5506, Part I, 883-890.

. Kilbas, A. A., Srivastava H. M., Trujillo, J. J., (2006). Theory and Application of Fractional Differential Equations, Elsevier Science, Amsterdam.

Delavari, H. et al., (2012). Stability Analysis of Caputo Fractional-Order Non-Linear Systems Revisited, NonLinear Dynamics, Vol. 67, No. 4, 2433-2439.

Bouchaud, J. P., Georges, A., (1990). Anomalous Diffusion in Disordered Media: Statistical Mechanisms, Models and Physical Applications, Phys. Rep., Vol. 195(4-5), 127-293.

Klimek, M., Agrawal, O. P., (2013). Fractional Sturm-Liouville Problem, Computers and Mathematics with Applications, Vol. 66, No. 5, 795-812.

Podlubny, I., (1999). Fractional Differential Equations, Mathematics in Science and Engineering, Academic Press, Vol. 198, San Diego, California, USA.

Zayernouri, M., Karniadakis, G. E., Fractional Sturm-Liouville Eigen-Problems, Theory and Numerical Approximation, Journal of Computational Physics, Vol. 252, 495-517.

Metzler, R., Klafter, J., (2002). The Random Walk's Guide to Anomalous Diffusion a Fractional Dynamics Approach, Phys. Rep., Vol. 339, No. 1, 67-90.

Bochner, S., Chandrasekharan, K., (1949). Fourier Transforms, Princeton University Press.

Blaszczyk, T., Ciesielski, M., (2014). Numerical Solution of Fractional Sturm-Liouville Equation in Integral Form, Fractional Calculus and Applied Analysis, Vol.17, No. 2, 307-320.

Mainardi, F., (2010). Fractional Calculus and Waves in Linear Viscoelasticity, An Introduction to Mathematical Models, World Scientific, Singapore, 368, 2010.

Ibrahim, R., Momani, S., (2007). On the Existence and Uniqueness of Solutions of a Class of Fractional Differential Equations, J. Math. Anal. Appl., Vol. 334, No. 1, 1-10. 
Tapdigoglu, R., (2019). Problemes inverses pour des equations differentielles aux derives fractionnaires, Docteur de thesis, Universite de la Rochelle, France.

Samko, S. G., (2003). Fractional Weyl-Riesz Integro Differentiation of Periodic Functions of Two Variables Via the Periodization of the Riesz Kernel, Appl. Anal. J., Vol. 82, No. 3, 269-299.

Pooseh, S., Rodrigues, H. S., Torres, D. F. M., (2011). Fractional Derivatives in Dengue Epidemics, AIP Conf. Proc., Vol. 1389, 739-742. 